\title{
Lettre à la rédaction : Soumission chimique par GHB : Cheveux et GC/MS/MS
}

\section{Pascal KINTZ, Vincent CIRIMELE, Carole JAMEY, Bertrand LUDES}

Institut de Médecine Légale, 11, rue Humann - F-67000 STRASBOURG - FRANCE

Tél : +33 390243349 - e-mail : pascal.kintz@wanadoo.fr

Le GHB est un produit naturellement présent chez l'homme à des concentrations nanomolaires.

Son sel de sodium, classé comme stupéfiant depuis le 5 mai 1999 est une poudre blanche, micro-cristalline, fortement hygroscopique, soluble dans l'eau et de saveur salée. On le rencontre sur le marché noir sous forme de liquide translucide, pouvant être parfois coloré, dont les concentrations sont très variables.

Sur le plan pharmacologique, le GHB fait partie de la classe des hypnotiques et anesthésiques, avec des indications comme inducteur de sommeil ou comme adjuvant des analgésiques et autres anesthésiques en chirurgie. La posologie usuelle est de $60 \mathrm{mg} / \mathrm{kg}$ chez l'adulte et $100 \mathrm{mg} / \mathrm{kg}$ chez l'enfant. Il produit hypnose et hypotonie, mais pas d'effet analgésique ou myorelaxant. L'administration IV provoque une augmentation modérée et transitoire de la tension artérielle, associée à une bradycardie et une diminution de la fréquence respiratoire. Le GHB est également un hypothermisant, un hypokaliémiant et un ocytocique (1).

La demi-vie du GHB est proportionnelle à la dose administrée, probablement due à une limitation de sa dégradation enzymatique. Par voie IV, elle est de 40 min pour une dose à $60 \mathrm{mg} / \mathrm{kg}$ (2), per os elle est de 20 à $25 \mathrm{~min}$ pour des doses de 12,5 à $50 \mathrm{mg} / \mathrm{kg}$ (3), avec un pic plasmatique atteint en 20 à $45 \mathrm{~min}$. Ces résultats sont conformes à ceux observés par Kintz et al (4) après administration orale d'une charge de GHB à 60 $\mathrm{mg} / \mathrm{kg}$, avec un pic plasmatique à $125 \mathrm{mg} / \mathrm{l}$ obtenu à 20 min. La même étude confirmait une élimination plasmatique complète en 5 heures.

La lecture de la littérature internationale démontre que le GHB peut essentiellement être observé dans 3 situations à implication médico-légale, la soumission chimique, le dopage sportif et enfin la conduite automobile.

A ce jour, il n'existe pas de test rapide d'identification du GHB. Aucun réactif d'immunochimie n'est disponible sur le marché, ce qui conduit très certainement à sous-estimer le nombre réel de sujets exposés à ce produit (5).

Par voie orale, le GHB est facilement absorbé et rapidement éliminé. La caractérisation dans l'urine semble impossible après 8 à 10 heures. Une formation in vitro dans le sang non conservé sur EDTA ou en post mortem, ainsi que des concentrations urinaires de l'ordre de quelques $\mathrm{mg} / \mathrm{l}$ peuvent en outre compliquer l'interprétation (6).

Kintz et al $(4,7)$ ont comparé l'excrétion du GHB dans la salive et la sueur par rapport au sang et aux urines après administration contrôlée d'une dose unique de 60 $\mathrm{mg} / \mathrm{kg}$, solubilisée dans 1 verre d'eau sucrée. La comparaison des différents milieux a montré que la salive 
ne permet pas d'augmenter la fenêtre de détection par rapport au sang, que l'élimination urinaire est très rapide et qu'enfin la sueur pourrait apporter des informations complémentaires, du fait de ses propriétés cumulatives. Néanmoins, ce dosage ne semble pas accessible en routine.

Dans le cadre d'une soumission par GHB, la tâche du toxicologue expert est rendue difficile par plusieurs paramètres : délais souvent importants entre les faits et les prélèvements, demi-vie courte, absence de réponse aux tests immuno-chimiques, analyse non réalisée en routine et concentrations physiologiques de l'ordre de 0,5 à $3 \mathrm{mg} / \mathrm{l}$, même lorsque les prélèvements sont faits dans les conditions optimales.

Les cheveux sont généralement présentés comme une matrice permettant la caractérisation d'une usage répété de xénobiotiques (8). Récemment, l'utilisation d'un couplage ultra-sensible (GC/MS/NCI) a permis de mettre en évidence une exposition unique à $2 \mathrm{mg}$ de flunitrazépam (9).

Sur cette base, le protocole qui suit a été mis en place pour documenter un acte de soumission chimique impliquant le GHB.

Il est impératif de prélever les cheveux 1 mois après les faits, afin de suivre, par segmentation, les variations de concentrations.

Les cheveux (une mèche d'au moins 100 cheveux) sont coupés au ras du cuir chevelu, dans des conditions strictes d'alignement, et maintenus par une cordelette. La conservation avant analyse se fait à température ambiante. Après décontamination par 2 bains successifs de $5 \mathrm{ml}$ de dichlorométhane pendant $2 \mathrm{~min}$, les cheveux sont coupés en segments de $0,3 \mathrm{~mm}$ sur une longueur de $3 \mathrm{~cm}$. Chaque segment $(5-10 \mathrm{mg})$ va ensuite être extrait selon la méthode suivante : incubation pendant 16 heures à $56^{\circ} \mathrm{C}$ dans $500 \mu 1$ de $\mathrm{NaOH} 0,01 \mathrm{M}$ en présence de $10 \mathrm{ng}$ de $\mathrm{GHB}-\mathrm{d}_{6}$ utilisé comme standard interne, neutralisation par $500 \mu \mathrm{l}$ de $\mathrm{HCl} 0,01 \mathrm{M}$, extraction par $3 \mathrm{ml}$ d'acétate d'éthyle en milieu acide $(100 \mu \mathrm{l}$ $\mathrm{H}_{2} \mathrm{SO}_{4} 0,01 \mathrm{M}$ ), dérivation par silylation par un mélange $20 \mu \mathrm{l}$ BSTFA $+1 \%$ TMCS et $20 \mu \mathrm{l}$ d'acétate d'éthyle, puis injection de $1 \mu 1$ dans une colonne HP5-MS (30 $\mathrm{m} \times 0,25 \mathrm{~mm}$ ) d'un système de chromatographie gazeuse (HP 5890 Série II) couplé à un détecteur de masse tandem (Finnigan TSQ 700) en mode impact électronique et SRM. Les ions parents du GHB $(\mathrm{m} / \mathrm{z} 233)$ et GHB- $\mathrm{d}_{6}(\mathrm{~m} / \mathrm{z} 239)$ sont filtrés par le premier quadripôle. Les ions fils communs m/z 147 et 148 du GHB et du GHB- $\mathrm{d}_{6}$ sont sélectionnés par le $3^{\text {ime }}$ quadripôle après collision avec l'argon à une pression de 0,62 mTorr sous une tension de $-8 \mathrm{~V}$ (offset).

La linéarité a été vérifiée de 0,2 à $20 \mathrm{ng} / \mathrm{mg}(\mathrm{r}=0,9987)$ pour un échantillon de $5 \mathrm{mg}$.

Les concentrations physiologiques varient de 0,5 à 12,0 ng/mg ( $n=24)$, sans corrélation en fonction de la couleur des cheveux. Ces résultats sont comparables à ceux de Frison et al (10), mais supérieurs à ceux de Gaillard et al, qui en outre ont observé une incorporation plus faible dans les cheveux blonds par rapport aux cheveux bruns ou noirs (11).

Dans ces conditions, il est illusoire de vouloir comparer des individus entre eux, sur la base de la concentration mesurée. Par contre, si on segmente les cheveux d'un même sujet, il est possible d'évaluer un profil d'exposition, en utilisant les concentrations de chaque segment comme référence. En effet, s'il n'y a pas exposition exogène, les concentrations de chaque segment doivent rester très proches. Au contraire, si les segments sont assez courts, par exemple de $0,3 \mathrm{~cm}$, une exposition unique doit conduire à une augmentation ponctuelle de la concentration, par rapport aux autres segments qui servent alors de contrôle (en particulier pour établir la concentration physiologique propre du sujet). Cette théorie a été vérifiée par Pépin et al (12) dans les poils de la barbe après une épreuve de surcharge.

Nous avons, dans un premier temps, établi un profil de concentration chez 2 sujets naifs, l'un avec des cheveux blonds, l'autre avec des cheveux noirs (Figure 1). Les concentrations apparaissent comme constantes sur la longueur étudiée, à l'exclusion du segment proximal, probablement reflet de l'incorporation sudorale qui n'aurait pas encore été totalement éliminée par les soins hygiéniques classiques.

Une expertise judiciaire, impliquant une agression sexuelle sous influence d'une substance psycho-active, d'une jeune fille de 19 ans nous a permis de confirmer l'intérêt de l'analyse du GHB dans les cheveux. La victime ayant décidé de porter plainte 5 jours après les faits, l'analyse du sang ou des urines était sans objet. Nous avons conseillé aux services de gendarmerie de convoquer la jeune fille pour un prélèvement de cheveux environ 1 mois après les faits, nous permettant ainsi d'encadrer la période du crime, sur la base d'une vitesse de pousse de l'ordre de $1 \mathrm{~cm} /$ mois. Les résultats obtenus (Figure 1) sont sans équivoque et démontrent donc une exposition ponctuelle au GHB. En effet, un pic de concentration est obtenu pour la période correspondant au crime sexuel.

En conclusion, la spectrométrie de masse tandem apparaît comme l'outil de référence pour documenter une soumission chimique par GHB. Sa sensibilité importante complétée par sa haute spécificité permet de mettre en évidence une exposition unique, à distance des faits. Chaque sujet étant son propre contrôle, toute variation de concentration sur la longueur de la mèche pourra être interprétée comme le témoin irréfutable d'une agression chimique. Il est indispensable d'attendre 1 mois entre les faits et le prélèvement, afin de documenter les concentrations physiologiques. 


\section{$7 \mathrm{ng} / \mathrm{mg}$}

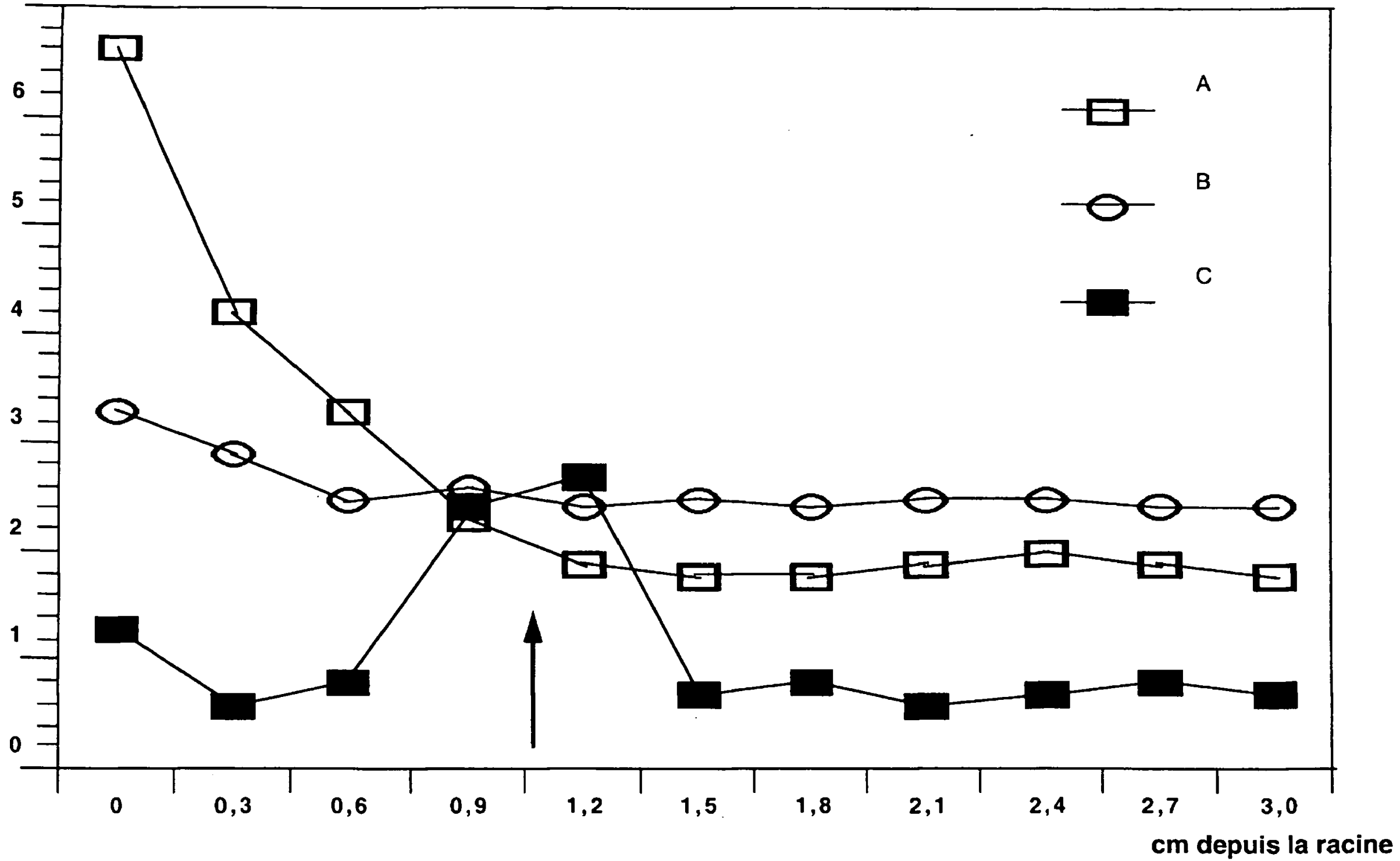

Figure 1 : Profil de concentrations en GHB chez 2 sujets témoins ( $A$ : cheveux blonds et $B$ : cheveux noirs) et une victime (C) d'une agression sexuelle sous influence.

\section{Références}

1. Ghysel M.-H. Le GHB : l'acide gamma hydroxybutyrique. Revue de la littérature. Toxicorama. $1999 ; 11: 1$ 11.

2. Vree T.B., Daars A.M., Van der kleijn E. Capacity-limited elimination of 4-hydroxybutyrate, ethanol and vinylbital. Pharm. Week. $1975 ; 110: 1257-62$.

3. Ferrara S.D., Zotti S., Tedeschi L. et al. Pharmacokinetics of gamma-hydroxybutyric acid in alcohol dependent patients after single and repeated oral doses. Br. J. Clin. Pharmacol. 1992 ; 45 : 353-6.

4. Kintz P., Goullé J.P., Cirimele V., Ludes B. Window of detection of GHB in blood and saliva. Clin. Chem. $2001 ; 47$ : 2033-4.

5. Deveaux M., Renet S., Renet V. et al. Utilisation de l'acide gamma-hydroxybutyrique (GHB) dans les raves-parties et pour la soumission chimique en France : mythe ou réalité ? Acta Clin. Belgica 2002 ; 57, suppl 1 : 37-40.

6. Kintz P. Difficultés analytiques et d'interprétation dans les expertises toxicologiques liées à la présence de GHB. J. Med. Leg. Droit Med. 2002, sous presse.

7. Kintz P., Villain M., Cirimele V., Goullé J.P., Ludes B. Usage criminel de substances psycho-actives : le problème de la durée de détection. Acta Clin Belgica. 2002 ; 57, suppl 1 : 24-30.
8. Goullé J.P., Kintz P. Un nouveau moyen d'investigation biologique : l'analyse des cheveux. Intérêt en pratique médicale. Rev. Med. Interne 1996 ; 17 : 826-35.

9. Negrusz A., Moore C.M., Hinkel K.B. et al. Deposition of 7-aminoflunitrazepam and flunitrazepam in hair after a single dose of Rohypnol. J. Forensic Sci. 2001 ; 46 : 1143-51.

10. Frison G., Tedeschi L., Brusini G., Castagna F., Ferrara S.D. GC/MS analysis of gamma-hydroxybutyrric acid (GHB) in human hair. 2nd international meeting on clinical and forensic aspects of hair analysis, 6-8 juin 1994, Genova.

11. Gaillard Y., Goullé J.P., Pépin G. Determination of naturally endogenous levels of GHB in human hair. Are there possibilities in the identification of GHB intake through hair analysis in case of rape under the influence of the drug ? J. Anal. Toxicol., soumis.

12. Pépin G., Gaillard Y., Cheze M. Soumission chimique : à propos de quelques cas. $40^{\text {eme }}$ congrès de toxicologie clinique, 24-27 mars 2002, Djerba. 